\title{
A DRUG-BASED INTERVENTION STUDY ON THE IMPORTANCE OF BUFFALOES FOR HUMAN SCHISTOSOMA JAPONICUM INFECTION AROUND POYANG LAKE, PEOPLE'S REPUBLIC OF CHINA
}

\author{
JIAGANG GUO, YUESHENG LI, DARREN GRAY, AN NING, GUANGHAN HU, HONGGEN CHEN, \\ GEORGE M. DAVIS, ADRIAN C. SLEIGH, ZHENG FENG, DONALD P. MCMANUS,* AND GAIL M. WILLIAMS \\ Institute of Parasitic Diseases, Chinese Centre for Disease Control and Prevention, Shanghai, People's Republic of China; Australian \\ Centre for International and Tropical Health and Nutrition, University of Queensland and Queensland Institute of Medical Research, \\ Brisbane, Queensland, Australia; Jiangxi Provincial Institute of Parasitic Diseases, Nanchang, People's Republic of China; \\ Department of Microbiology and Tropical Medicine, George Washington University Medical Center, Washington, District of \\ Columbia; National Centre for Epidemiology and Population Health, Australian National University, Canberra, \\ Australian Capital Territory, Australia
}

\begin{abstract}
Schistosomiasis japonica is a zoonosis of major public health importance in southern China. We undertook a drug intervention to test the hypothesis that buffalo are major reservoirs for human infection in the marshlands/lake areas, where one million people are infected. We compared human and buffalo infection rates and intensity in an intervention village (Jishan), where humans and buffalo were treated with praziquantel, and a control village (Hexi), where only humans were treated, in the Poyang Lake region. Over the four-year study, human incidence in Jishan decreased but increased in Hexi. Adjustment of incidence by age, sex, water exposure, year, and village further confirmed the decreased human infection in Jishan. Chemotherapy for buffaloes resulted in a decrease in buffalo infection rates in Jishan, which coincided with the reduction in human infection rates there in the last two years of the study. Mathematical modeling predicted that buffalo are responsible for $75 \%$ of human transmission in Jishan.
\end{abstract}

\section{INTRODUCTION}

Reassessment of schistosomiasis-related disability, ${ }^{1}$ combined with recent information on the global prevalence of schistosome infection, ${ }^{2}$ indicates that the true burden of schistosomiasis is substantially greater than previously appreciated. Schistosomiasis japonica, which is caused by infection with Schistosoma japonicum, is a zoonosis that remains a serious public health problem in China, with approximately one million people infected. ${ }^{3,4}$ Locations with high endemicity occur around Dongting and Poyang Lakes, ${ }^{5-7}$ where the bulk of transmission occurs. ${ }^{4}$

We tested the hypothesis that water buffaloes are responsible for the persistence of human schistosomiasis in the lake areas, ${ }^{6}$ where they occur in large numbers and have a high infection rate and large stool mass, much of which is deposited in or near lake water. Despite descriptions of the importance of bovines in $S$. japonicum transmission, ${ }^{3,8}$ proof of this hypothesis had not been established. Results obtained were applied to a mathematical model of the dynamics of $S$. japonicum transmission ${ }^{9}$ to examine the predicted transmission in a scenario in which the buffalo contribution to human transmission was eliminated.

\section{METHODS}

Study areas. We selected Jishan and Hexi administrative villages in Poyang Lake area based on their similar schistosome transmission features that included a historical infection prevalence, transmission ecology (lake water height, flooding patterns), water contact patterns, buffalo/human numbers, herding practices, and sanitation facilities. ${ }^{6,10,11}$ Jishan is located on Jishan island (area $=5 \mathrm{~km}^{2}$ ) and has four natural

\footnotetext{
* Address correspondence to Donald P. McManus, Molecular Parasitology Laboratory, Queensland Institute of Medical Research, 300 Herston Road, Brisbane, Queensland 4006, Australia. E-mail: donM@qimr.edu.au
}

villages. Hexi (area $=3.7 \mathrm{~km}^{2}$ ) is located on Hexilong island and has six natural villages. The main daily activities of residents in both villages involve agriculture and fishing.

Study design. The basic study design has been reported. ${ }^{6}$ Jishan was selected as the intervention village because of its higher human prevalence at baseline. A medical questionnaire was administered to all study participants at baseline (pre-treatment) in late 1998. Questions included sociodemographic background, disease history, and history of lake water contact. A stool survey was conducted in both villages of 285 individuals with a high risk of schistosomiasis (any person reporting on the questionnaire any water contact in Poyang Lake). Each pre-treatment stool examination used two KatoKatz smears ${ }^{12}$ obtained from one stool specimen per person. All residents $\geq 5$ years of age in both villages were then treated (in early 1999) with praziquantel. One month after the first treatment, the fixed cohort of 285 individuals was checked again in both villages (one stool sample, two smears). Any individual still infected (at least one positive smear) was re-treated and re-checked one month later. No subject required a third treatment. As a result, we effectively demonstrated that all in the fixed cohort from both villages were free of schistosome infection (May 1999) before the start of the ongoing longitudinal study of incidence.

All humans and buffaloes in Jishan were mass treated annually with praziquantel (40 $\mathrm{mg} / \mathrm{kg}$ for humans and $25 / \mathrm{mg} / \mathrm{kg}$ for buffaloes), but only humans in Hexi received annual mass treatment. In 2001 and 2002, treatment of buffaloes was increased to twice per year. In both villages, human chemotherapy coverage was at least $97 \%$ throughout the study. In the first year of study, buffalo chemotherapy coverage was $87 \%$. Subsequently, the coverage was increased, reaching $>98 \%$ in the last two years of the study. At the end of each year from 1999 to 2002 all villagers who were resident were requested to provide a stool sample (three Kato-Katz smears) for measuring the $S$. japonicum annual human incidence (defined as the proportion of infected persons) and infection intensity (geometric mean eggs per gram (of feces [epg]). 
Stools freshly collected from all buffaloes were tested using the miracidial hatching test (MHT). ${ }^{13}$ Once a buffalo was identified as positive (miracidia observed hatching), another portion of the original stool sample was subjected to a rigorous microscopic egg count procedure to determine infection intensity (epg). ${ }^{13}$ Approximately 200 residents were randomly selected from each village and asked to complete an activity diary of their water contact. ${ }^{14}$ One day per week over an-eight week period in each transmission period they were asked to record the duration (in minutes) and site of water contact, and body part exposed for that day and the previous day. They were followed throughout the course of the study (over eight transmission periods in the spring and autumn of each year). The duration time of water contact was totaled annually for each person. Sampling methods for oncomelanid intermediate host snails have been reported, ${ }^{7}$ and collections were made in April and November of each year of the study.

Statistical analysis. Data were entered in Epi-Info version 6.01. ${ }^{15}$ Analysis was performed using Epi-Info and SAS software (SAS Institute, Cary, NC). Presence of human infection was defined as at least one egg in all Kato-Katz smears. Egg counts were transformed to epg and geometric mean intensity was calculated by using the log-transformed egg counts. Confidence intervals (CIs) were calculated using standard formulas based on the binomial distribution (annual incidence of infection) and the lognormal distribution (intensity). Presence of buffalo infections was defined as a positive MHT result. Infection intensity was calculated as for humans. Density of infected snails was calculated as the number of infected snails per $100 \mathrm{~m}^{2}$, and prevalence of snail infections as the proportion of infected snails.

Formal analyses of human and buffalo infection rates used a generalized linear model (GLM) with a logit link and a binomial error distribution. Analyses of intensities used a GLM (with actual egg counts per stool as the dependent variable) with a log link and negative binomial error distribution. Generalized equation estimators of parameters with an unstructured variance-covariance matrix were used to account for repeated measures on individuals over time. The base model included year (from 1999 to 2002), village, and a yearvillage interaction. The interaction parameters represent differential effects between villages over time and provided a test of the intervention effect. Effects were then adjusted for sex, age, and water contact. The model was used to obtain adjusted annual incidences. Analyses used the GENMOD procedure of SAS software.

Adjustment of intervention effects was effected by grouping duration into four groups: 0 minutes, 1-10 minutes, 11-20 minutes, and $>20$ minutes. This variable was used as a covariable and as part of a second-order interaction with village and year. Analyses that incorporated water contact were restricted to the smaller cohort of 200 persons.

Buffalo infections were analyzed using the same methods as for human infections. Snail infections were analyzed using the negative binomial model applied to the number of infected snails, and with a total number of snails as offset in the case of the snail prevalence. Since each year represented a new site sample, repeated measures were not taken into account.

Mathematical modeling. A mathematical model has been developed to simulate the transmission of schistosomiasis, and enable the prediction of the effect of interventions. ${ }^{9}$ This published model extends the two-host model of Barbour ${ }^{16}$ to allow for heterogeneity within the two major classes of definitive hosts. It consists of a set of simultaneous equations that model rate of change in prevalence over time. The model was then parameterized according to schistosomiasis epidemiology within study villages, including the distribution of endemic prevalences within host classes, and known features of schistosomiasis infection such as infection duration. Interventions are then imposed on the system, and the equations are solved numerically to predict the consequences for prevalence and incidence. The model was implemented within MATHCAD software. ${ }^{17}$

Ethical approval. Written ethical approval for this study was obtained from the national, provincial and village levels within China, and approval for the study was granted by the National Institutes of Health (United States) prior to commencement. Study participants identified as stool egg-positive for schistosomiasis were treated with $40 \mathrm{mg} / \mathrm{kg}$ of praziquantel, the current dosage recommended by the World Health Organization. Oral informed consent was obtained from all adults and from parents or guardians of minors who were involved in the study.

\section{RESULTS}

Study site characteristics at baseline. The baseline prevalence of $S$. japonicum in villagers was $20.3 \%(95 \%$ CI = $15.6-24.9 \%)$ in Jishan $(\mathrm{n}=773)$ and $10.9 \%(95 \% \mathrm{CI}=7.3-$ $14.4 \%$ ) in Hexi ( $\mathrm{n}=1,073)$, indicating a two-fold excess of infection (Figure 1a) (crude odds ratio $[\mathrm{OR}]=1.91$ ). However, the odds ratio pre-trial adjusted for water contact from fishing and previous treatment was 1.33 . The baseline prevalence of $S$. japonicum in buffalo was $9.9 \%$ (95\% CI $=4.3$ $15.5 \%)(\mathrm{n}=665)$ in Jishan and $12.4 \%(95 \% \mathrm{CI}=6-18.6 \%)$ in Hexi $(n=364)$ (Figure $1 c)$. The mean \pm SD age of residents in Jishan and Hexi villages at baseline was $29.9 \pm 15.5$ and $28.1 \pm 15.7$ years, respectively. The sex ratios (males:females) at baseline in Jishan and Hexi were 417:356 and 577: 496, respectively. The percentage of village residents treated for schistosomiasis in the two years before baseline was $31 \%$ in Jishan and $38 \%$ in Hexi.

Human incidence and intensity. Human S. japonicum incidence and intensity of infection are shown in Figure $1 \mathrm{a}$ and $\mathrm{b}$. In 1999, the incidence for Hexi (control)) was $4.2 \%$ (95\% CI $=2.5-5.6 \%)$, and it increased over the four-year study period to $10.3 \%(95 \% \mathrm{CI}=7.3-13.2 \%)$ in 2002 . The incidence in Jishan (intervention) was $8.9 \%(95 \% \mathrm{CI}=6.3-11.5 \%)$ in the first year of the study, and it decreased to $5.6 \%(95 \% \mathrm{CI}=$ $3.1-7.5 \%$ ) over the four-year study period.

A two-fold excess of baseline infection prevalence was seen in Jishan compared with Hexi in the first year of the study. However, over the study period this trend changed, resulting in Hexi having a two-fold excess of infection compared with Jishan.

In Jishan, males had a higher incidence (13.1\% versus $4.7 \%$ in $1999 ; P=0.001$ and $7.8 \%$ versus $3.2 \%$ in $2002 ; P=0.069$ ) than females. In Hexi, however, males had a significantly higher incidence only at the end of study $(4.9 \%$ versus $3.4 \%$ in $1999 ; P=0.38$ and $15.1 \%$ versus $4.7 \%$ in $2002 ; P=$ 0.0006).

The intensity of $S$. japonicum infection decreased significantly in Jishan from 1.14 epg $(95 \% \mathrm{CI}=0.8-1.6 \mathrm{epg})$ at 
(a) Human Infection Prevalencellncidence

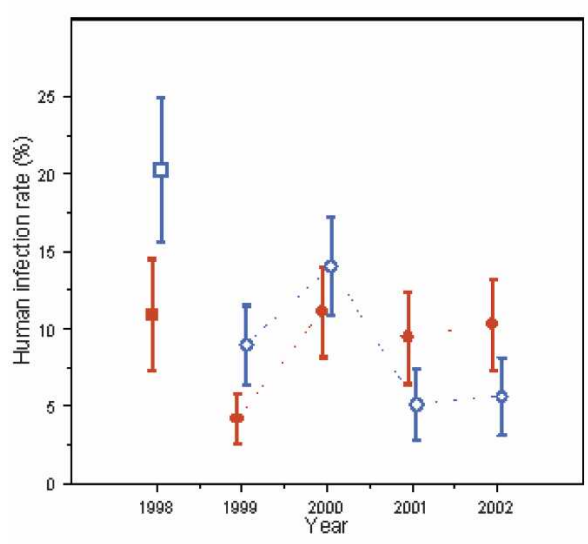

(c) Buffalo Infection Rate

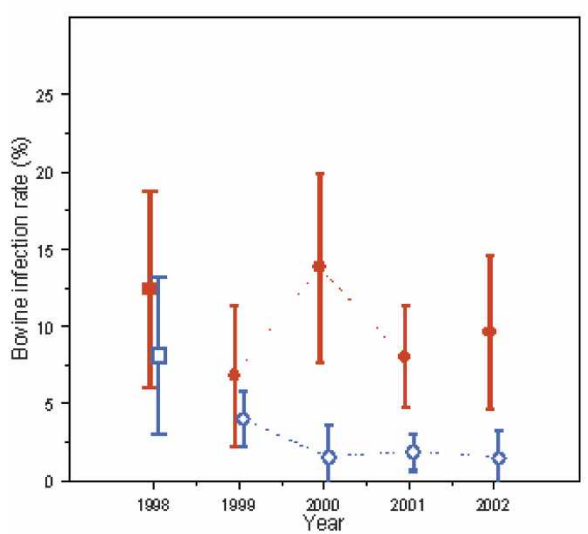

(e) Snail Infection Prevalence

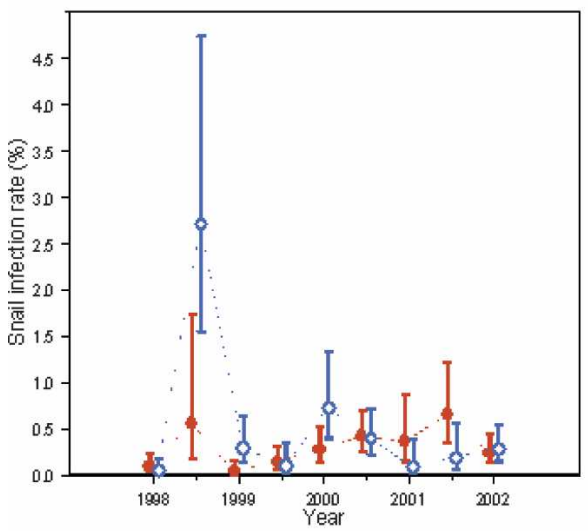

(b) Human Infection Intensity

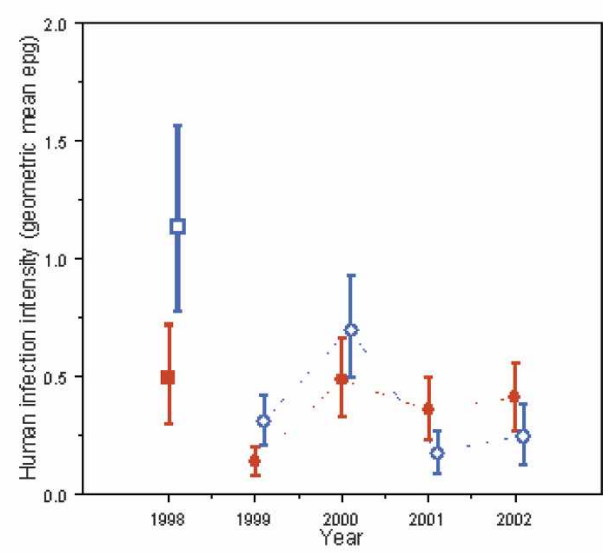

(d) Buffalo Infection Intensity
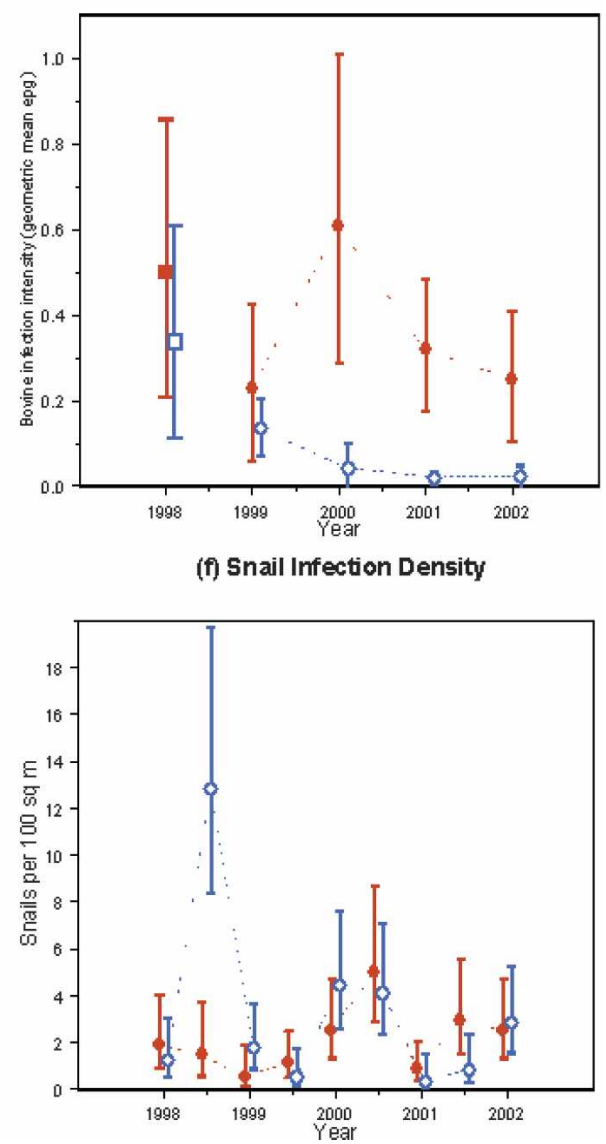

Figure 1. Human, buffalo, and snail infections in the intervention and control villages, Poyang Lake, People's Republic of China, $1998-2002$. The first two points in panels a-d represent prevalence and the remaining points represent incidence. $=$ Hexi; $\bigcirc=$ Jishan. epg $=$ eggs per gram (of feces). Error bars show 95\% confidence intervals. This figure appears in color at www.ajtmh.org.

baseline to 0.25 epg $(95 \% \mathrm{CI}=0.1-0.4 \mathrm{epg})$ in 2002 . However, the geometric mean intensity of infection in humans was relatively stable with a baseline value of $0.50 \mathrm{epg}(95 \% \mathrm{CI}=$ $0.3-0.7 \mathrm{epg}$ ) compared with $0.41 \mathrm{epg}(95 \% \mathrm{CI}=0.3-0.6 \mathrm{epg})$ in 2002.

Human infection adjusted for age, sex, water contact and year. Water exposure (1999-2002) in Jishan was 5.0 minutes/ day/person/year and 9.0 minutes/day/person/year in Hexi. Compared with the water exposure in the first year (1999), the duration of water contact increased by $39.8 \%$ and $34.8 \%$ in Jishan and Hexi, respectively, in 2002. Adjustment for age and sex rusted in little change in the annual incidences (Table $1)$, with the interaction parameters being significant for 2001 and 2002 ( $P=0.0004$ and $P=0.0005$, respectively). This indicated significant intervention effects in those years. Adjustment for water contact reduced the significance of the effect ( $P=0.012$ for 2001 and 0.024 for 2002), although this reduction was largely the result of loss of power because this analysis was restricted to a cohort of approximately 200 persons. The relative reduction in human incidence after adjust- 
TABLE 1

Incidence (\%) adjusted by age, sex, and water exposure in Jishan (intervention) and Hexi (control) villages

\begin{tabular}{lrrrrrrr}
\hline & \multicolumn{3}{c}{ Incidence (\%)* } & & \multicolumn{3}{c}{ Incidence (\%) $\dagger$} \\
\cline { 2 - 3 } Year & Jishan & \multicolumn{1}{c}{ Hexi } & $P$ & & Jishan & Hexi & $P$ \\
\hline 1999 & 8.95 & 4.17 & & & 7.74 & 4.02 & \\
2000 & 14.20 & 11.05 & 0.1317 & & 19.25 & 13.75 & 0.602 \\
2001 & 4.90 & 9.17 & 0.0001 & & 4.81 & 9.24 & 0.0117 \\
2002 & 5.60 & 10.25 & 0.0001 & & 6.97 & 15.54 & 0.0243 \\
\hline * Incidence (\%) adjusted by age and sex. & & & \\
† Incidence (\%) adjusted by age, sex, and water exposure.
\end{tabular}

ments for age, sex, and water contact was $48 \%$ in 2001 and $55 \%$ in 2002. More detailed examination of infection rates and water contact over time showed that water contact was higher in Hexi than in Jishan, particularly in the final season (autumn 2002), and that water contact was associated with human infection in Hexi, but not in Jishan (Figure 2a). Overall, the relationship between infection and water contact was such that risk of infection increased linearly up until an exposure of 20 minutes per day, after which the risk of infection plateaued (Figure 2b). This suggests that if infection is to occur, it will occur within 20 minutes of cercarial exposure.

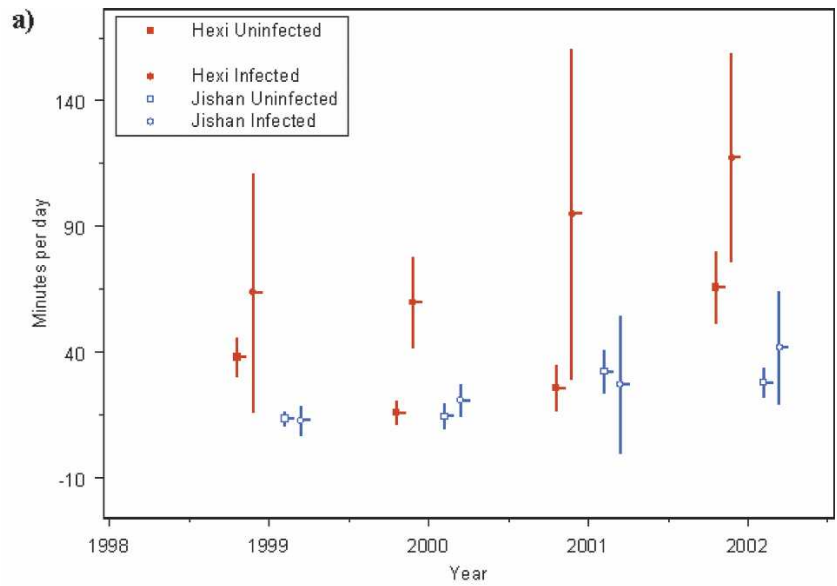

b)

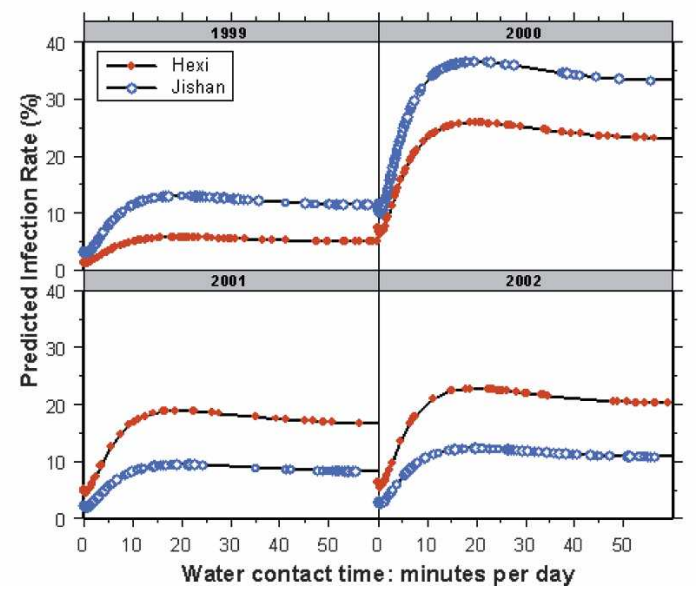

FIgURE 2. Water contact and Schistosoma japonicum infection. a, Water contact by year, village, and infection status, Poyang Lake, People's Republic of China, 1999-2002. Error bars show 95\% confidence intervals. b, Predicted human infection rates according to water contact in the intervention and control villages. This figure appears in color at www.ajtmh.org.
Buffalo infection rate and infection intensity. The results of the buffalo $S$. japonicum infection rate and intensity of infection are shown in Figure 1c and d. In the first year of the study (1999), the infection rate for Hexi was $7.6 \%$ (95\% CI = $2.8-12.4 \%)$ and it increased slightly to $9.6 \%(95 \% \mathrm{CI}=4.6-$ $14.6 \%)$ over the four-year study period in 2002. Comparatively, the infection rate in Jishan was $4.5 \%(95 \% \mathrm{CI}=2.6$ $6.4 \%$ ) in the first year of the study, and it decreased to $1.7 \%$ (95\% CI $=-0.2-3.6 \%)$ over the four-year study period.

A similar trend to that observed in humans occurred in the buffalo. As the overall annual infection rate increased in Hexi, it decreased in Jishan. The annual infection rate increased considerably in 2000. In Hexi, the infection rate increased to $13.8 \%(95 \% \mathrm{CI}=7.7-20.0 \%)$ in 2000 before decreasing to $8 \%(95 \% \mathrm{CI}=4.7-11.3 \%)$ in 2001 . In Jishan, however, an increase was not seen. The infection rate decreased to $1.5 \%(95 \% \mathrm{CI}=-0.6-3.7 \%)$ in 2000 before increasing to $1.9 \%(95 \% \mathrm{CI}==0.7-3.1 \%)$ in 2001 .

There was a highly significant decrease in the intensity of infection in Jishan from 0.34 epg $(95 \% \mathrm{CI}=0.1-0.6 \mathrm{epg})$ at baseline to $0.02 \mathrm{epg}(95 \% \mathrm{CI}=0-0.05 \mathrm{epg}$ ) at the end of the study. Similarly, there was a significant decrease in the intensity of infection in Hexi from the baseline value of $0.50 \mathrm{epg}$ (95\% CI $=0.2-0.9 \mathrm{epg})$ to $0.25 \mathrm{epg}(95 \% \mathrm{CI}=0.1-0.4 \mathrm{epg})$ in 2002 (Figure 1d).

Estimates of the role of buffalo in transmission. A total of 2,092 buffaloes were examined in Hexi and Jishan from late 1998 to 2002. A mean prevalence of $5.6 \%$ (males $=8.5 \%$, females $=4.5 \%)$ was observed. Younger buffaloes (6-11 months of age) in both Jishan and Hexi had a significantly higher $(P=0.007)$ prevalence $(12.2 \%)$ and mean intensity of infection (3.8 epg) than older animals ( $>3$ years or 36 months of age), which had a prevalence of $1.8 \%$ and a mean intensity of infection of $0.49 \mathrm{epg}$ (Figure 3). Most (76.3\%) of the total egg output from all buffalo examined was from animals less than 12 months of age.

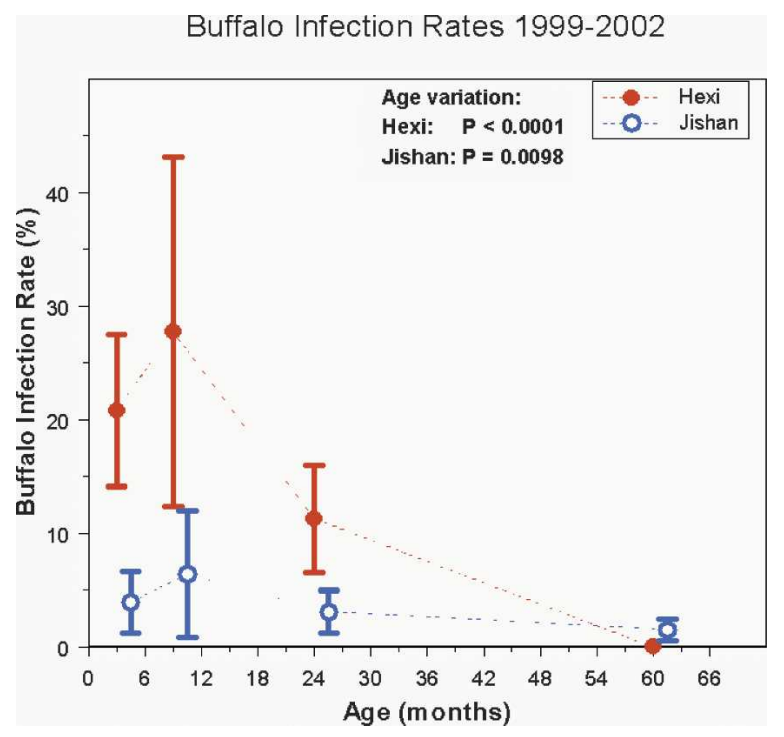

FIGURE 3. Buffalo infection rate adjusted by age in the intervention and control villages, Poyang Lake, People's Republic of China, 1999-2002. Error bars show 95\% confidence intervals. This figure appears in color at www.ajtmh.org. 
Using baseline endemic prevalence and estimated treatment efficacy and coverage, our model was used to predict the trial outcome in Jishan and to compare it with that actually obtained (Figure 4). This appeared to be a good fit, except for 2000, which was an aberrant point in both villages. To estimate the transmission caused by buffaloes, the removal of buffalo transmission was simulated by curing all buffaloes at the start of an intervention period, and simultaneously vaccinating them with a $100 \%$ effective vaccine. This simulation showed that incidence of infection in humans was reduced immediately from $20 \%$ to approximately $5 \%$, with a slow decrease thereafter, which amounted to a reduction in infections of $75 \%$. Therefore, we conclude that buffaloes are responsible for $75 \%$ of human transmission.

Dynamics of infected snails. The prevalence and density of infected snails over nine seasons showed non-significant differences between the villages, with the exception of the unusual spike of infection in Jishan in the spring of 1999 (Figure 1e and f). There were also parallel increases in the density of infected snails in the fall of 2000 and the spring of 2001 and a subsequent decrease in the fall of 2001.

\section{DISCUSSION}

This drug-based intervention showed a two-fold excess of infection in humans in Jishan (intervention village) compared with Hexi (control village) at baseline and in the first year of the study. At the end of the study, the human infection rate in Jishan was half that of Hexi; thus, Hexi showed a two-fold excess of infection compared with Jishan. A large reduction in the buffalo infection rate in Jishan over the course of the study also occurred, decreasing to $1.7 \%$ in 2002. This coincided with the reduction in human infection rates in Jishan in the last two years of the study. It suggested that buffalo chemotherapy impacted on the human incidence because of the removal of buffalo as a transmission factor by blocking of environmental contamination with buffalo feces containing schistosome eggs.

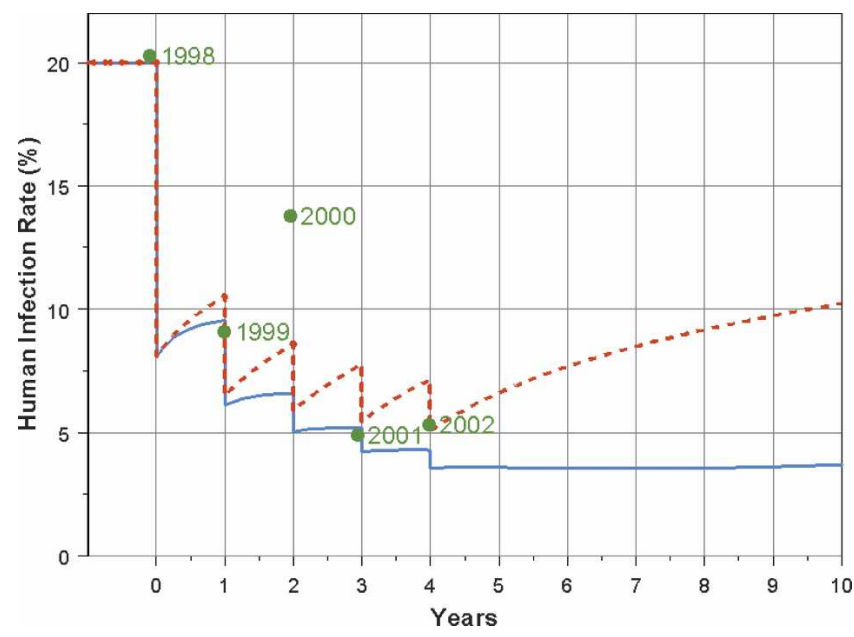

Figure 4. Human infection prevalence in Jishan, People's Republic of China predicted from the mathematical model of Williams and others. ${ }^{9}$ Dashed line $=$ human treatment only; solid line $=$ human and buffalo treatment combined. Dots show the prevalences actually observed in Jishan. The gap between the two curves represents predicted prevented infections associated with buffalo treatment. This figure appears in color at www.ajtmh.org.
However, a direct link between buffalo infection rates and human incidence could not be established because the pattern of snail infection was not consistent with this interpretation. It should be noted that snail infection density, not prevalence, is the important factor in determining the impact of snail infection on human infection. Prevalence is dependent on the density of miracidia and the overall number of snails in an area, while human infection rates depend on the density of cercariae, which are determined by the number of infected snails per unit area. We would expect the density of infected snails to decrease along with both the human and buffalo infection rates. The density of infected snails fluctuated from season to season, but the difference between Hexi and Jishan was not significant in 2001-2002. Village-wide data are not the critical data needed to measure exposure at individual levels because snail infection may be subjected to sudden environmental changes, for example, droughts or floods. In 2001, snails were driven underground because of a prolonged drought, thus making them uncollectable on the marshlands, and lowering estimated density of infection.

In the absence of a direct link between buffalo chemotherapy and the reduction in human incidence because of the snail infection rates, we need to consider alternative explanations for the significant relative decrease in incidence in Jishan. In China, it is estimated that up to 40 species of wild and domestic animals are infected with schistosomes, but many of these are not considered to act as reservoirs. ${ }^{18,19}$ In the lakes region, the environmental contamination is largely due to water buffalo, particularly their high fecal output laden with schistosome eggs. Pigs, dogs, and rodents play a very small part, if any, in environmental contamination or transmission. This is because dogs are notably absent in these areas, pigs are confined to pens within the communities, and rodents are not believed to be a factor. ${ }^{7}$

The two villages were comparable because they were selected based on their similar ecologic features related to schistosome transmission, although at baseline there were more buffalo in Jishan (665 animals) than in Hexi (364). Although Jishan had a higher prevalence than Hexi (20\% versus $11 \%$, respectively), the relative difference in infection rates showed an odds ratio of 1.33 that was adjusted for water contact from fishing and previous treatment. Thus, we conclude that the areas were similar in relation to their infection rates. They also had similar age, sex, and history of schistosomiasis control.

Formal adjustment for the potentially major confounding factors of age, sex, and water contact did not significantly change the results. Although human infection rates are expected to decrease temporarily with human treatment, ${ }^{20}$ this effect would be expected to be the same in both villages. This was not observed in Hexi, indicating that human treatment did not materially influence human re-infection. The mathematical model applied to Jishan data demonstrates that human treatment reduces human infection rates, which then increase. Buffalo treatment is predicted to prevent a major component of this increase. In 2001 and 2002, infection rates in Hexi increased while the reduction in Jishan was maintained as predicted.

Having eliminated the major determinants of schistosomiasis transmission, it is difficult to posit the existence of an additional factor, which may have led to the specific reduction in Jishan. Buffalo fecal contamination of lake-marshland ar- 
eas has been empirically estimated as $>70 \%,{ }^{3}$ and it is biologically plausible that elimination of this contamination should reduce human and buffalo re-infection. Thus, buffalo chemotherapy was responsible for the reduction in human infection in Jishan by the prevention of an increase in the human infection rates. Therefore, buffalo are the major reservoir hosts for schistosomiasis transmission to humans in the lake regions.

We noted that the incidence of infection in residents in 2000 was increased in both villages (Figure 1). This could have been the result of an influx of new buffalo that resulted in increased schistosome transmission. At the end of 2000 and thereafter, we provided treatment to all newborn animals and buffalo recently imported into Jishan. The infection rate in humans decreased markedly from $14.0 \%$ in 2000 to $5.1 \%$ in 2001. This occurred even though 240 imported or newly born buffalo were recorded that year, making up more than half of the buffalo population in Jishan. The incidence in Jishan was controlled at a stable level of approximately $5 \%$, which contrasted with the incidence in Hexi, which remained high.

Current control programs in most schistosomiasis-endemic areas of China involve the provision of annual praziquantel treatment to infected persons. However, the infection rates still remain at relatively high levels because of re-infection. ${ }^{5,20}$ The results of this study provide evidence that buffaloes are major reservoirs for human schistosomiasis transmission in the lake regions of China and that buffalo chemotherapy is effective at reducing both bovine and human infections and re-infections. We are currently undertaking a cluster-randomized bovine intervention trial with increased power in two Chinese provinces to test for general applicability.

The removal of bovines as a schistosomiasis transmission factor through chemotherapy also provides the rationale for development of a transmission-blocking vaccine applicable to buffalo. Bovine chemotherapy with numerous mass treatments per year is very expensive compared with a bovine vaccine that would probably only need to be administered once or twice. If such a transmission-blocking vaccine can be developed and put into practice in combination with other control strategies such as human chemotherapy, elimination of $S$. japonicum is possible. ${ }^{21}$

Received July 18, 2005. Accepted for publication September 2, 2005.

Acknowledgments: We thank all staff at the Jiangxi Provincial Institute of Parasitic Diseases-Chinese Centre for Disease Control and Prevention and other local provincial staff who assisted with the extensive field work.

Financial support: This study was supported by the National Institute of Allergy and Infectious Diseases Tropical Medicine Research Center grant 1 P 50AI-39461) and a National Health and Medical Research Council (NHMRC) of Australia and Wellcome Trust (United Kingdom) International Collaborative Research Grants Scheme Award. Darren Gray is a NHMRC Postgraduate Public Health Scholar.

Disclosure: None of the authors has any conflicts of interest.

Authors' addresses: Jiagang Guo, Guanghan Hu, and Zheng Feng, Institute of Parasitic Diseases, Chinese Centre for Disease Control and Prevention Chinese Academy of Preventive Medicine, 207 Rui Jin Er Lu, Shanghai 200005, People's Republic of China. Yuesheng Li, Darren Gray, Donald P. McManus, and Gail M. Williams, Australian Centre for International and Tropical Health and Nutrition, University of Queensland and Queensland Institute of Medical Research, 300 Herston Road, Brisbane, Queensland 4029, Australia. An Ning and Honggen Chen, Jiangxi Provincial Institute of Parasitic
Diseases, Nanchang, People's Republic of China. George M. Davis, Department of Microbiology and Tropical Medicine, George Washington University Medical Center, Washington, DC 20037. Adrian C. Sleigh, National Centre for Epidemiology and Population Health, Australian National University, Canberra, Australian Capital Territory 0200 , Australia.

Reprint requests: Donald P. McManus, Molecular Parasitology Laboratory, Queensland Institute of Medical Research, 300 Herston Road, Brisbane, Queensland 4006, Australia, Telephone: 61-7-3362-0401, Fax: 61-7-3362-0104, E-mail: donM@qimr.edu.au.

\section{REFERENCES}

1. King CH, Dickman K, Tisch DJ, 2005. Reassessment of the cost of chronic helmintic infection: a meta-analysis of disabilityrelated outcomes in endemic schistosomiasis. Lancet 365: $1561-1569$.

2. van der Werf MJ, de Vlas SJ, Brooker S, Looman CW, Nagelkerke NJ, Habbema JD, Engels D, 2003. Quantification of clinical morbidity associated with schistosome infection in sub-Saharan Africa. Acta Trop 86: 125-139.

3. Ross AG, Sleigh AC, Li Y, Davis GM, Williams GM, Jiang Z, Feng Z, McManus DP, 2001. Schistosomiasis in the People's Republic of China: prospects and challenges for the 21st century. Clin Microbiol Rev 14: 270-295.

4. Zheng J, 2003. Schistosomiasis control in China: progress and challenges. Chin J Parasitic Dis Parasitol 21: 4-5.

5. Li YS, Sleigh AC, Ross AGP, Williams GM, Tanner M, McManus DP, 2000. Epidemiology of Schistosoma japonicum in China: Morbidity and strategies for control in the Dongting Lake of region. Int J Parasitol 30: 273-281.

6. Guo JG, Ross AG, Lin DD, Williams GM, Chen HG, Li Y, Davis GM, Feng Z, McManus DP, Sleigh AC, 2001. A baseline study on the importance of bovines for human Schistosoma japonicum infection around Poyang Lake, China. Am J Trop Med Hyg 65: 272-278.

7. Davis GM, Wu WP, Chen HG, Liu HY, Guo JG, Lin DD, Lu SB, Williams G, Sleigh A, Feng Z, McManus DP, 2002. A baseline study of importance of bovines for human Schistosoma japonicum infections around Poyang lake, China: villages studied and snail sampling strategy. Am J Trop Med Hyg 66: 359-371.

8. He YK, Zhou SJ, Wu ZW, 1991. Role of farm cattle, water buffaloes and pigs in transmission of schistosomiasis japonica in the Dongting Lake region. Chin J Schistosomiasis Control 3: 44-45.

9. Williams GM, Sleigh AC, Li Y, Feng Z, Davis GM, Chen H, Ross AG, Bergquist R, McManus DP, 2002. Mathematical modelling of schistosomiasis japonica: comparison of control strategies in the People's Republic of China. Acta Trop 82: 253-262.

10. Zhang S, Liu Z, Li G, Zhong J, Cheng Y, 1990. Snail distribution and susceptible zones of schistosomiasis in endemic areas around Poyang Lake. Chin J Parasitic Dis Parasitol 81: 8-12.

11. Davis GM, Wilke T, Zhang Y, Xu X, Qiu CP, Spolsky CM, Qiu D, Li YS, Xia MY, Zheng F, 1999. Snail-Schistosoma, Paragonimus interactions in China: population ecology, genetic diversity, coevolution and emerging diseases. Malacologia 41: 355-377.

12. Katz N, Chaves A, Pellegrino J, 1972. A simple device for quantitative stool thick-smear technique for schistosomiasis mansoni. Rev Inst Trop Sao Paulo 14: 397-400.

13. Chen MG, 1989. Schistosomiasis control programme in the People's Republic of China: a review. Southeast Asian J Trop Med Public Health 20: 511-517.

14. Ross AG, Yuesheng L, Sleigh AC, Williams GM, Hartel GF, Forsyth SJ, Yi L, McManus DP, 1998. Measuring exposure to Schistosoma japonicum in China. I. Activity diaries to assess water contact and comparison to other measures. Acta Trop 71: 213-228.

15. Epi-Info Version 6.01, 1994. Atlanta, GA: Centers for Disease Control and Prevention, Atlanta, GA and Geneva: World Health Organization.

16. Barbour A, 1996. Modelling the transmission of schistosomiasis: an introductory view. Am J Trop Med Hyg 55 (Suppl 5): 135143. 
17. Mathcad, 2004. Version 11.1. Cambridge, MA: Mathsoft Engineering and Education, Inc.

18. World Health Organization, 1993. The control of schistosomiasis: second report of a WHO Expert Committee. World Health Organ Tech Rep Ser 830: 1-86.

19. Johansen MV, Bøgh HO, Nansen P, Christensen NO, 2000. Schistosoma japonicum infection in the pig as a model for human schistosomiasis japonica. Acta Trop 76: 85-99.

20. Li YS, Sleigh AC, Ross AG, Li Y, Williams GM, Tanner M,
McManus DP, 2000. Two-year impact of praziquantel treatment for Schistosoma japonicum infection in China: reinfection, subclinical diseases and fibrosis marker measurements. Trans R Soc Trop Med Hyg 94: 191-197.

21. McManus DP, Wong JY, Zhou J, Cai C, Zeng Q, Smyth D, Li Y, Kalinna BH, Duke MJ, Yi X, 2002. Recombinant paramyosin (rec-Sj-97) tested for immunogenicity and vaccine efficacy against Schistosoma japonicum in mice and water buffaloes. Vaccine 20: 870-878. 